\title{
Economic effect between surgery and thermal ablation for patients with papillary thyroid microcarcinoma: a systemic review and meta-analysis
}

\author{
Shuqian Chen ${ }^{1} \cdot$ Yaqian $M a o^{1} \cdot$ Gang Chen $\mathbb{1}^{1,2}$
}

Received: 9 November 2021 / Accepted: 19 January 2022 / Published online: 5 February 2022

(c) The Author(s), under exclusive licence to Springer Science+Business Media, LLC, part of Springer Nature 2022

\begin{abstract}
Background At present, there are still many controversies regarding the treatment of papillary thyroid microcarcinoma (PTMC). It is worth noting that thermal ablation has become a viable alternative for patients at high risk of surgery or those who wish to receive more active treatment than active surveillance.

Objective To investigate the economic benefits, efficacy, and safety of thermal ablation for patients with PTMC.

Methods We searched PubMed, Cochrane Library, Web of Science, and Embase for articles published from the database establishment to August 2021. All of the studies included were retrospective cohort analyses. In addition, meta-analysis was performed using the Stata15.1 versions and Review Manager5.3. Data were extracted according to the PRISMA guidelines by two professional doctors.

Results A total of 7 articles (1582 patients) were included in our systematic review and meta-analysis. We discovered that thermal ablation could shorten the hospitalization time (SMD, $-3.82[95 \% \mathrm{CI},-5.25$ to -2.40$]$ ), operation time (SMD, $-5.67[95 \% \mathrm{CI},-6.65$ to -4.68$]$ ), cost (SMD, -3.40 [95\%CI, -5.06 to -1.75$]$ ) and reduce postoperative complications ( $\mathrm{OR}, 0.07$ [ $95 \% \mathrm{CI}, 0.01$ to 0.44$]$ ) than surgical treatment. In addition, our study also found that compared with surgery, there was no significant difference in the recurrence rate (OR, 1.53 [95\% CI, 0.63 to 3.73]) and metastasis rate (OR, 0.47 [95\% CI, 0.12 to 1.86$])$.

Conclusion On the premise of being safe and effective, thermal ablation has better economic benefits than surgical treatment for patients with PTMC.
\end{abstract}

Keywords PTMC $\cdot$ Thermal ablation $\cdot$ Economic effect $\cdot$ Safety $\cdot$ Efficacy

\section{Introduction}

Thyroid micropapillary carcinoma is the maximum size of thyroid papillary carcinoma (PTC) at no more than $1 \mathrm{~cm}$ $[1,2]$. Since the popularization of physical examination consciousness and the continuous updating of ultrasound equipment, the incidence of PTMC has been gradually increased [3, 4].

Gang Chen

chengangfj@163.com

1 Shengli Clinical Medical College of Fujian Medical University, Fuzhou 350001 Fujian, China

2 Department of Endocrinology, Fujian Provincial Hospital, Fuzhou 350001 Fujian, China
Nowadays, the social economy has been paid more attention in the medical field. One of the problems is to reduce medical costs while ensuring the safety and effectiveness of treatment. The epidemic of COVID-19 has seriously impacted the world's economy. Therefore, it is urgent to enhance medical support and optimize medical costs in this situation.

Thyroid surgery is currently the standard treatment for PTMC, but it has scars and complications side effects, while higher medical costs are inevitable. Active surveillance is mentioned in the 2015 ATA guidelines for very low-risk PTMC. However, the indications and contraindications are not very clear. Some people may develop anxiety during the active surveillance process [5, 6]. According to joint guidelines published in the European Thyroid Journal, mainly if the patient is at surgical risk, or is unwilling to undergo surgery or active surveillance, the patient can 
consider the use of thermal ablation for patients with lowrisk PTMC [7]. Multicenter studies in many countries have shown that thermal ablation (radiofrequency ablation, RFA; microwave ablation, MWA; and laser ablation, LA) is an effective and safe alternative for patients with high surgical risk or refusing surgery $[5,6,8,9]$.

The purpose of this meta-analysis is to compare variables between surgery and thermal ablation, such as hospital stay, operation time, cost, recurrence rate, metastasis rate, and complication rate, to prove the economic effects, safety, and effectiveness of thermal ablation.

\section{Materials and methods}

\section{Literature retrieval strategy}

To compare the safety, effectiveness, and economic effects of surgery and thermal ablation, we searched the relevant articles published in PubMed, Web of Science, Embase, and Cochrane Library from the establishment of the database to August 2021. In addition, we reviewed the reference list of the retrieved articles to identify potentially relevant studies further. The search strategy included MeSH terms and corresponding synonyms for thyroid papillary microcarcinoma, surgery, and thermal ablation.

\section{Inclusion and exclusion criteria for related studies}

Inclusion criteria:(1) no larger than $10 \mathrm{~mm}$ in diameter, and no lymph node metastasis or distant metastasis; (2) all subjects in the experimental group were treated with thermal ablation, while those in the control group were treated with surgery (lobectomy or total thyroidectomy); (3) the experimental methods used in the study were randomized controlled trials or retrospective analysis; (4) the experimental results included hospital stay, operation time, cost, recurrence rate, metastasis rate, and complication rate.

Exclusion criteria: if one of the following is excluded: (1) when overlapping populations are described in different studies, only studies with desired outcome indicators are included, while other studies are excluded; (2) if the outcome indicators needed are not described in the study, or the full text cannot be found, even contacting the author of the literature through various means of communication, such as e-mail, etc., and requesting to borrow detailed original data.

\section{Data extraction}

The following characteristics were extracted from the selected studies: author, sample size, region, study period, average tumor volume, hospital stay, operation time, cost, and clinical indicators of patients at the end of intervention (recurrence rate, metastasis rate, and incidence of complications). It is to evaluate the difference between thermal ablation intervention and surgical treatment. The data were extracted by two co-authors (Shuqian Chen and Yaqian Mao), and any differences of opinion were resolved through discussion. Other co-authors reviewed the extracted information to verify its accuracy.

\section{Literature quality assessment}

The included studies were assessed with the NewcastleOttawa scale (NOS).

\section{Statistical analysis}

Statistical analysis was conducted with Review Manager 5.3 , while binary variables were analyzed by calculating the odds ratio (OR) with a $95 \%$ confidence interval $(95 \%$ $\mathrm{CI})$. For continuous results, SMD is used as the amount of effect, and the corresponding $95 \% \mathrm{CI}$ is calculated. For all analyses, $P<0.05$ is considered to be statistically significant. The $\mathrm{I}^{2}$ test evaluates heterogeneity, and $\mathrm{I}^{2}>50 \%$ indicates significant heterogeneity. When there is significant heterogeneity, the random effect model (DerSimonian-Laird method) is used to calculate the combined effect; otherwise, the fixed model (Mantel-Haenszel method) is used instead. When there is significant heterogeneity, sensitivity analysis is used to evaluate the stability of the results. The Egger test of STATA15.1 version was used to assess possible publication bias. The funnel chart is not performed since the number of studies included in each result is less than 10 .

\section{Results}

The whole process of literature collection and screening is shown in Fig. 1. A total of 725 related articles was collected by searching the database. After 27 review articles and 57 case reports were excluded, we deleted 583 records by looking at the title and abstract. As a result, only 58 articles need to be reviewed. Because the result of Lan et al's study is quality of life, there are few studies on quality of life, so this study is not included. Finally, seven articles met the selection criteria. The main features of the study are summarized in Table 1 . The results of the meta-analysis are shown in Figs. 2-7. And egger's funnel plots are shown in the Fig. 8. Seven articles were searched, and a total of 1582 patients was included in the systematic review and meta-analysis. All articles are observational studies, and the number of studies about RFA, MWA and LA was four, two and one, respectively. All the subjects were from China, mostly from economically developed areas. 
Fig. 1 Procedure for the selection process

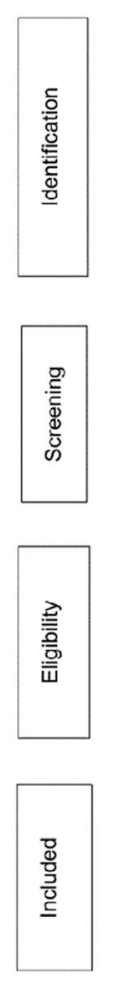

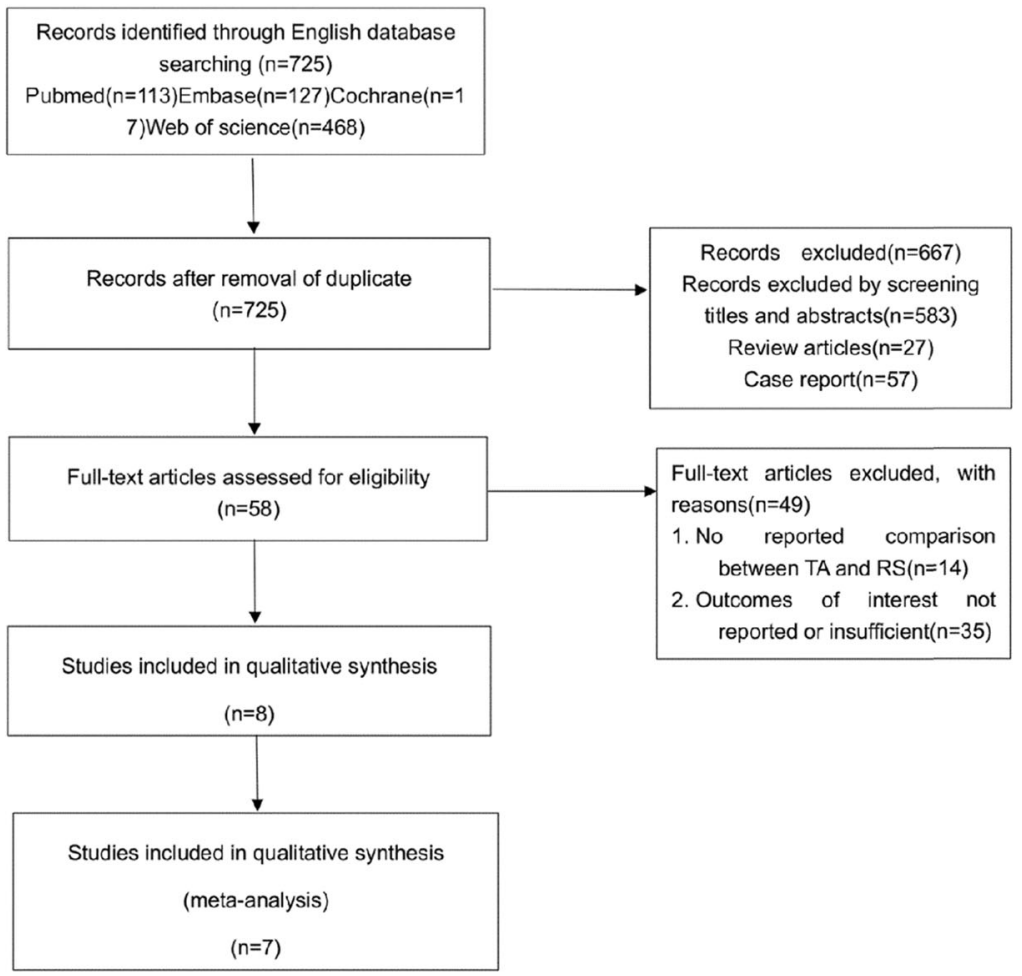

The included study used the NOS to evaluate the quality. The results showed that the overall quality of NOS was high. Five studies [10-14] mentioned the metastasis rate and cost, six studies [10-15] reflected the recurrence rate, seven studies [10-16] related to the incidence of complications, hospital stay, and operation time.

In terms of hospital stay $\left(P<0.0001, \mathrm{I}^{2}=96 \%, \mathrm{SMD}=\right.$ $-3.82,95 \% \mathrm{CI}-5.25$ to -2.40$)$ and operation time $(P<$ $0.00001, \quad \mathrm{I}^{2}=94 \%, \quad \mathrm{SMD}=-5.67,95 \% \mathrm{CI}-6.65$ to -4.68), thermoablation group was shorter than surgery group. It is confirmed by Egger test that the result of hospital stay may have publication offset $(P=0.04)$ and the result of operation time don not have publication offset $(P$ $=0.774)$. In terms of cost, 1414 samples were involved, and the results were statistically significant $\left(P<0.0001, \mathrm{I}^{2}=\right.$ $99 \%, \quad \mathrm{SMD}=-3.40,95 \% \mathrm{CI}-5.06$ to -1.75$)$, which showed the cost of thermal ablation was less than that of the surgical group. The sensitivity analysis showed that the results were reliable, and the Egger test confirmed that there may be a publication offset $(P=0.044)$. In terms of recurrence rate, 1495 samples were involved, and the results were not statistically significant $\left(P=0.34, \mathrm{I}^{2}=0 \%, \mathrm{OR}=\right.$ $1.53,95 \% \mathrm{CI} 0.63$ to 3.73 ). In terms of transfer rate, 1414 samples were involved, and the results were not statistically significant $\left(P=0.28, \mathrm{I}^{2}=0 \%\right)$. The combined results were $\mathrm{OR}=0.47,95 \% \mathrm{CI} 0.12$ to 1.86 . Regarding the incidence of complications, there was significant difference between patients who underwent surgery and those who underwent thermal ablation $\left(P=0.005, \mathrm{I}^{2}=83 \%, \mathrm{OR}=\right.$ $0.07,95 \%$ CI 0.01 to 0.44 ). We conducted sensitivity analysis to analyze the causes of heterogeneity, and the results suggested that the study of Song et al had a greater impact on heterogeneity. Excluding the study of Song et al (2021), $\mathrm{I}^{2}$ decreased from $83 \%$ to $31 \%$.

\section{Discussion}

Although active surveillance has been advocated for lowrisk PTMC patients [17], many patients reluctant to accept active surveillance and choose surgery or ablation treatment caused by anxiety and psychological stress of diagnosed PTMC [18]. Because PTMC is an inert tumor, on the premise of being safe and effective, the medical treatment cost performance should be considered. High cost and long hospital stay of surgical treatment are unavoidable. Besides, it is also possible to cause complications associated with surgery, such as hoarseness and permanent hypothyroidism. The quality of life after surgery might be even lower than that before operation [19]. According to the existing research, the quality of patients' life in the ablation group was better than the surgery group [20]. And the morbidity associated with surgical intervention was inversely associated with physician experience. 


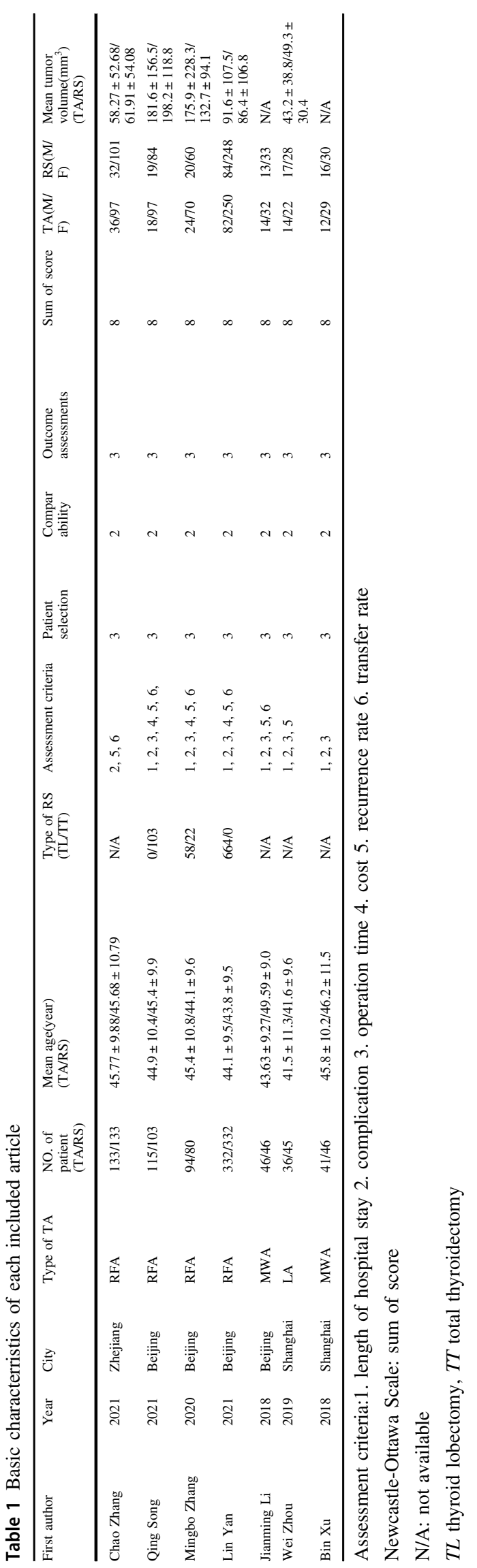

Papillary carcinomas in the isthmus tend to show multiple foci and capsular invasion, total thyroidectomy seems to be more appropriate than less-than-total thyroidectomy [21]. The site of PTMC studied by Song et al (2021) is located in the isthmus, without metastasis lymph nodes and no gland outside invasion, total thyroidectomy and thermal ablation can be chosen for PTMC. This study showed hypothyroidism complication rates of total thyroidectomy are higher than thermal ablation, and thermal ablation has a better quality of life and economic effect. There is no obvious difference in recurrence rate and transfer rate between them.

Current treatment aims to cure the disease at the lowest cost. With the increase of spending on health care and the limited medical resources, we are forced to optimize medical resources. We should consider the economic impact of interventions, and choose the most suitable treatment for patients $[22,23]$. A cost-benefit analysis assesses the relative value of health care intervention [24]. The inclusion of cost in clinical decision-making does not mean that cost is the only consideration, which contributes to patients' participation in self-regulation [25], and achieves the lowest mortality and morbidity with the lowest treatment burden [26]. For individuals, the cost is an essential consideration in seeking medical care. For society, cost types can be broken down into three main components: direct costs associated with using health resources, indirect costs related to lost productivity, and sometimes intangible costs (cost associated with pain) [27]; reducing costs leads to better allocation of resources. Especially during the COVID-19 pandemic, the pandemic has taken a significant toll on the economy. In the current social situation, it is necessary to consider the cost problem and optimize the maximum return $[28,29]$.

Compared with surgery, the cost of thermal ablation, length of hospital stay, and operating time are shorter. For patients, lower health care costs are directly related to personal health choices. Furthermore, for society, shorter hospitalization time and operation time are better for the redistribution of medical resources, and it can maximize health benefits to patients within available resources [30].

In PTMC with no evidence of extra thyroid expansion and lymph node metastasis, thermal ablation has become the main treatment for primary low-risk PTMC [31-33]. Compared with conventional surgery, thermal ablation significantly reduced the volume of primary tumor, had fewer postoperative complications, had a lower incidence of other serious complications, avoided complications related to general anesthesia, and achieved rapid recovery [5, 32, 34]. Although PTMC is an indolent tumor with a good prognosis, recurrence rate and metastasis rate over a longer follow-up period are more significant. Prospective studies from China suggested that no residual or recurrent 


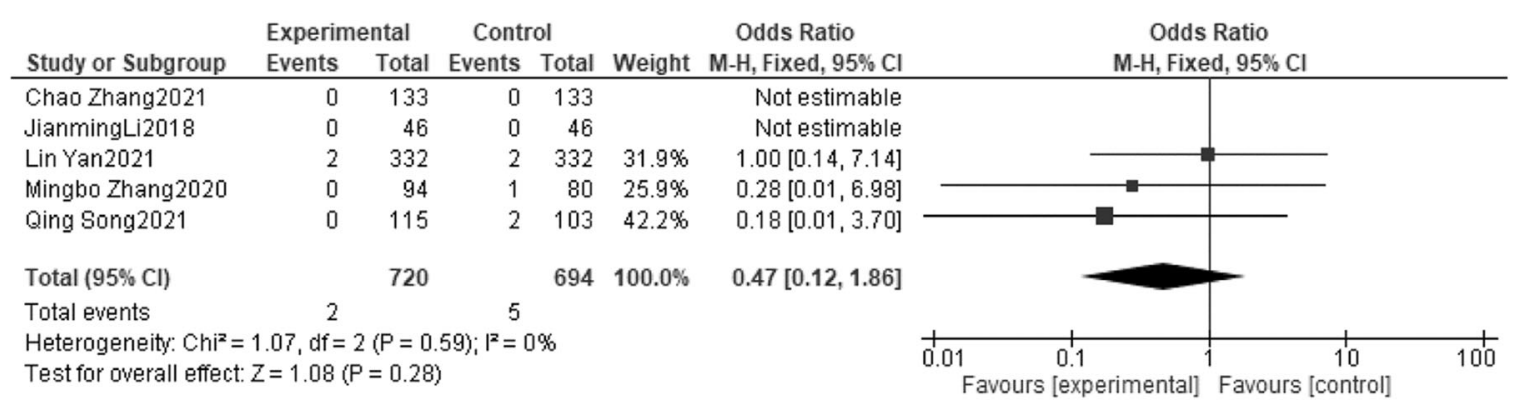

Fig. 2 Forest plot transfer rate

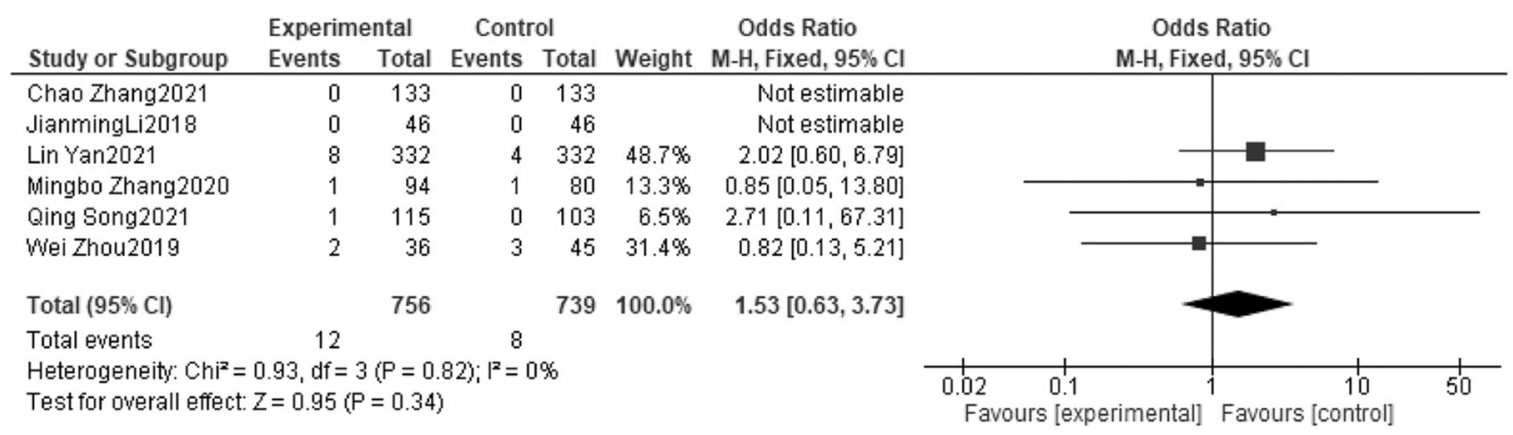

Fig. 3 Forest plot the recurrence rate

\begin{tabular}{|c|c|c|c|c|c|c|c|c|c|c|}
\hline Study or Subgroup & \multicolumn{2}{|c|}{ Experimental } & \multicolumn{2}{|c|}{ Control } & Weight & $\begin{array}{c}\text { Odds Ratio } \\
\text { M-H, Random, } 95 \% \mathrm{Cl}\end{array}$ & \multicolumn{4}{|c|}{$\begin{array}{c}\text { Odds Ratio } \\
\text { M-H, Random, } 95 \% \mathrm{Cl}\end{array}$} \\
\hline Bin Xu2018 & 2 & 41 & 7 & 46 & $15.9 \%$ & $0.29[0.06,1.46]$ & & $\Longrightarrow$ & & \\
\hline Chao Zhang2021 & 3 & 133 & 5 & 133 & $16.3 \%$ & $0.59[0.14,2.52]$ & & & & \\
\hline Jianming Li2018 & 2 & 46 & 20 & 46 & $16.1 \%$ & $0.06[0.01,0.27]$ & & & & \\
\hline Lin Yan2021 & 0 & 332 & 14 & 332 & $12.8 \%$ & $0.03[0.00,0.56]$ & & & & \\
\hline Mingbo Zhang2020 & 0 & 94 & 3 & 80 & $12.4 \%$ & $0.12[0.01,2.30]$ & & & & \\
\hline Qing Song2021 & 2 & 115 & 103 & 103 & $12.3 \%$ & $0.00[0.00,0.00]$ & $\leftarrow$ & & & \\
\hline Wei Zhou2019 & 1 & 36 & 3 & 45 & $14.2 \%$ & $0.40[0.04,4.02]$ & & & & \\
\hline Total $(95 \% \mathrm{Cl})$ & & 797 & & 785 & $100.0 \%$ & $0.07[0.01,0.44]$ & & & & \\
\hline Total events & 10 & & 155 & & & & & & & \\
\hline $\begin{array}{l}\text { Heterogeneity: } \mathrm{Tau}^{2}= \\
\text { Test for overall effect }\end{array}$ & $\begin{array}{l}5.14 ; \mathrm{Chi}^{2} \\
\mathrm{Z}=2.80(\mathrm{~F}\end{array}$ & $\begin{array}{l}=34.90, \\
=0.005\end{array}$ & $d f=6(P$ & $<0.00$ & 001); $\left.\right|^{2}=$ & & $\begin{array}{r}0.001 \\
\mathrm{Fa}\end{array}$ & $\begin{array}{c}0.1 \\
\text { vours [experimental] }\end{array}$ & $\begin{array}{c}10 \\
\text { Favours [control] }\end{array}$ & 1000 \\
\hline
\end{tabular}

Fig. 4 Forest plot the complication rate

tumor tissue was found during the long-term follow-up of PTMC patients with RFA [35]. And studies from the United States with follow-up of more than 4 years also showed that PTMC patients who underwent ablation had no recurrence or metastasis of PTMC during follow-up [36, 37]. Ultrasound-guided thermal ablation is a novel and minimally invasive candidate technique for the management of thyroid lesions. Several ablation guidelines and consensus statements indicate that thermal ablation can be used for benign and selective malignant thyroid nodules [38]. RFA may be an alternative strategy for the treatment of PTMC
[39]. The thyroid tissue injury caused by PLA is transient and self-limited [40]. Several trials have demonstrated that PLA is technically feasible for complete destruction of PTMC [41]. All patients can well tolerate this procedure and tolerate ablation under local anesthesia without the need for analgesics [36]. MWA has been proved to be safe and effective [42].

In 2017 RFA guideline of Korean society of thyroid radiology, it was accepted that RFA can be used for patients with primary thyroid cancer who refuse surgery or who cannot undergo an operation $[43,44]$. The results of this 


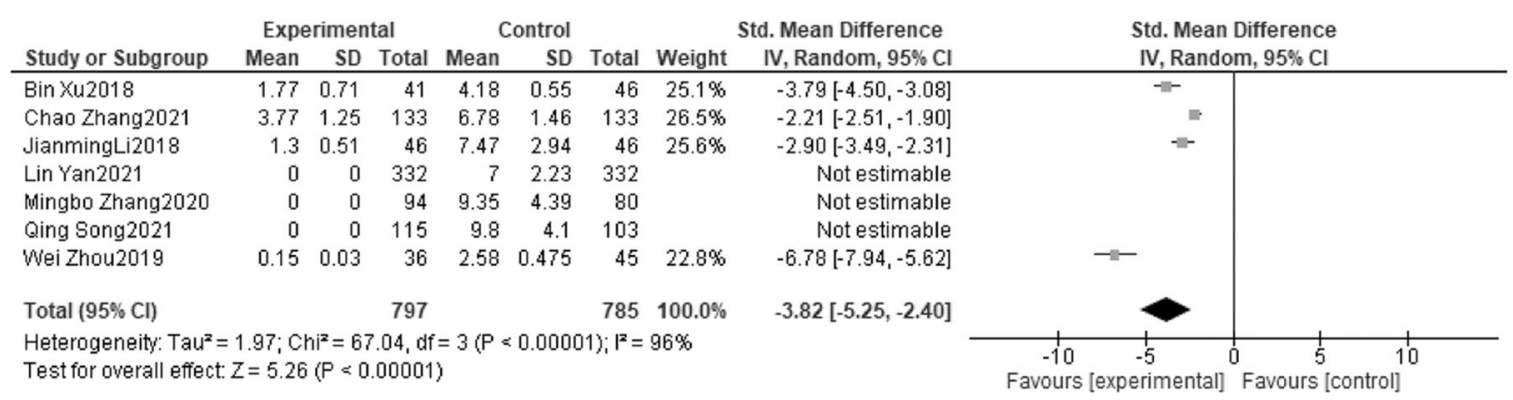

Fig. 5 Forest plot hospital stay

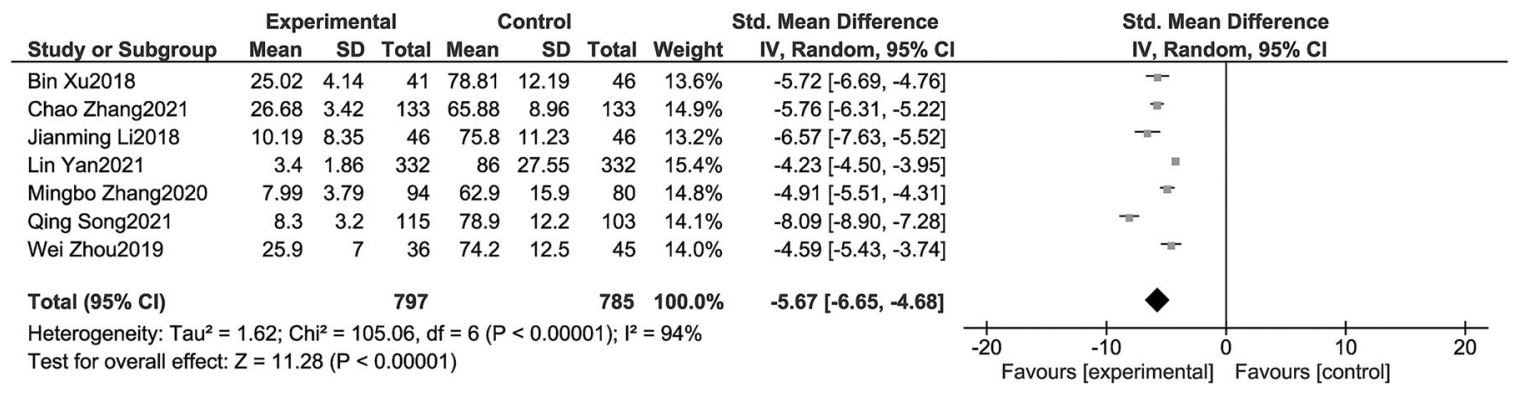

Fig. 6 Forest plot the operation time

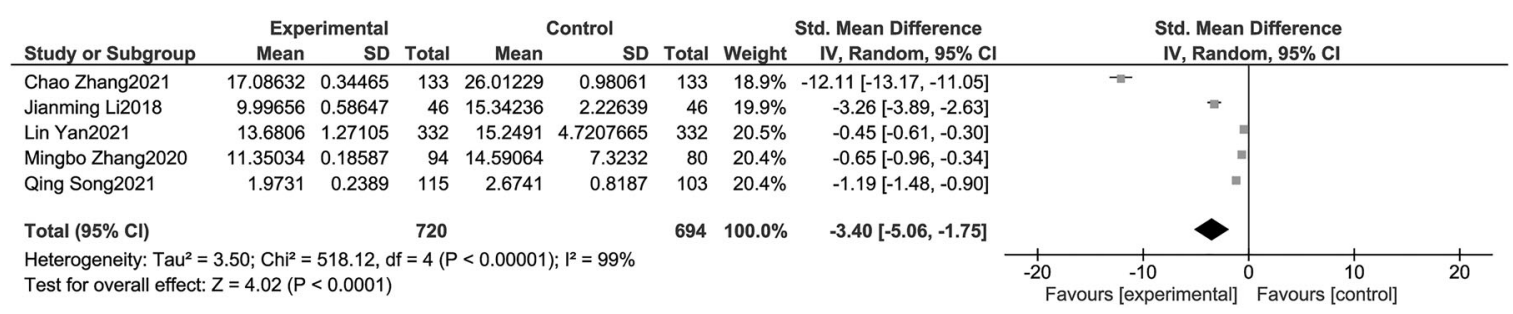

Fig. 7 Forest plot the cost. The unit of cost is thousand yuan. Experimental means TA, and control means RS

meta-analysis suggest that surgery has no obvious advantage over thermal ablation in reducing metastasis rate and recurrence rate. And in terms of hospital stay, operation time and the cost, thermal ablation technique is significantly shorter than surgery. And thermal ablation reduces postoperative complications. Several studies have shown that ablation is an effective and safe treatment for PTMC [3, 4, 6, 19, 34, 45-48]. Reducing the length of hospital stay also contributes to the redistribution of health resources. It means that thermal ablation is more in line with socioeconomic impacts. At present, most of the thermal ablation technologies are carried out in some areas, and due to limited equipment and ultrasound technology in some areas, such technologies have not yet been implemented.

The content about thermal ablation has not been included in the current guidelines. However, studies have proved that thermal ablation is economical, safe, and effective, and the part about thermal ablation may be added into the update of the guidelines in the future [49].

\section{Limitations}

First, PTMC is a disease with slow progress. Thermoablation is a new technology developed in recent years. At present, the follow-up time after treatment is not long enough, and the long-term safety and effectiveness of its efficacy are not evaluated. Second, the included studies are all from China, so it should be better to have studies from other countries and different ethnic groups to explore whether the results are consistent. Third, all the included studies are retrospective studies, and more randomized controlled trials are needed to examine this issue in the 

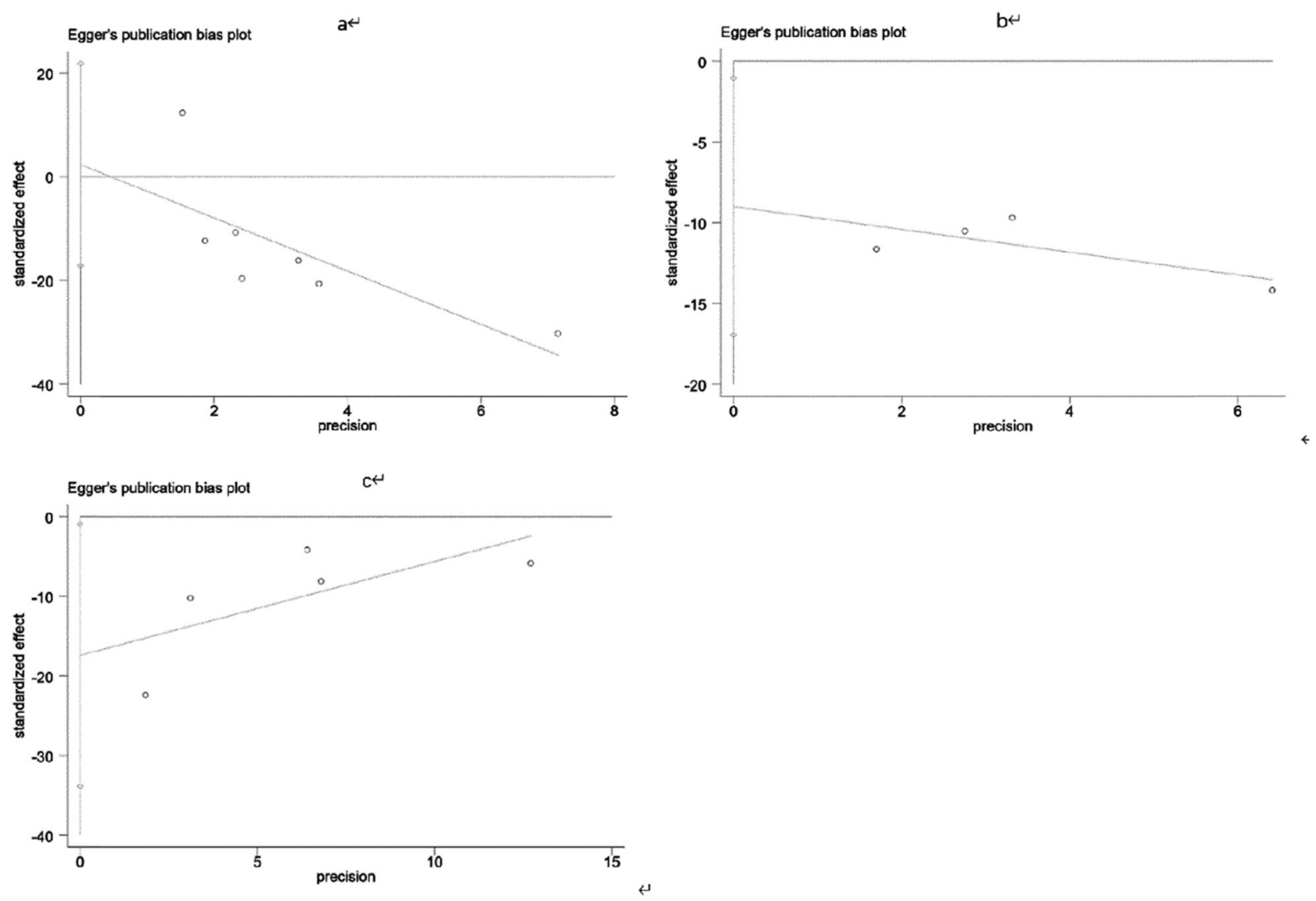

Legend

- regression line

$\diamond \diamond: 95 \%$ CI for intercept

Fig. 8 Egger's funnel plot of (a) complication rate; (b) length of stay; (c) the cost during hospitalization

future. Finally, we never get final pathology on the tumor by ablation; Fine Needle Aspiration (FNA) is limited in that results are inconclusive and the sample is not always representative of the whole tumor.

The results of our systematic review and meta-analysis show that the thermal ablation is more economical.

Acknowledgements We wish to thank all the patients and their family members that participated in the study.

Author contributions S.C. and G.C. designed the study; S.C. and Y.M. acquired data; S.C. wrote the manuscript; G.C. analyzed the clinical data and revised the manuscript.

\section{Compliance with ethical standards}

Conflict of interest The authors declare no competing interests.

Publisher's note Springer Nature remains neutral with regard to jurisdictional claims in published maps and institutional affiliations.

\section{References}

1. K. Kim, X. Zheng, J.K. Kim et al. The contributing factors for lateral neck lymph node metastasis in papillary thyroid microcarcinoma (PTMC). Endocrine 69, 149-156 (2020)

2. Y. Ito, A. Miyauchi, H. Oda, Low-risk papillary microcarcinoma of the thyroid: A review of active surveillance trials. Eur. J. Surg. Oncol. 44, 307-315 (2018)

3. M. Ding, X. Tang, D. Cui et al. Clinical outcomes of ultrasoundguided radiofrequency ablation for the treatment of primary papillary thyroid microcarcinoma. Clin. Radio. 74, 712-717 (2019)

4. X.W. Bo, F. Lu, H.X. Xu et al. Thermal ablation of benign thyroid nodules and papillary thyroid microcarcinoma. Front Oncol. 10, $580431(2020)$

5. H. He, Q. Song, Y. Lan et al. Efficacy and safety of ultrasoundguided radiofrequency ablation for low-risk papillary thyroid microcarcinoma in patients aged 55 years or older: a retrospective study. Int. J. Hyperth. 38, 604-610 (2021)

6. L. Yan, Y. Lan, J. Xiao et al. Long-term outcomes of radiofrequency ablation for unifocal low-risk papillary thyroid microcarcinoma: a large cohort study of 414 patients. Eur. Radio. 31, 685-694 (2021)

7. G. Mauri, L. Hegedüs, R.L. Cazzato et al. Minimally invasive treatment procedures have come of age for thyroid malignancy: 
the 2021 clinical practice guideline for the use of minimally invasive treatments in malignant thyroid lesions. Cardiovasc Interv. Radio. 44, 1481-1484 (2021)

8. G. Mauri, F. Orsi, S. Carriero et al. Image-guided thermal ablation as an alternative to surgery for papillary thyroid microcarcinoma: preliminary results of an italian experience. Front Endocrinol. (Lausanne) 11, 575152 (2020)

9. G. Mauri, L. Hegedüs, S. Bandula et al. European Thyroid Association and Cardiovascular and Interventional Radiological Society of Europe 2021 Clinical Practice Guideline for the Use of Minimally Invasive Treatments in Malignant Thyroid Lesions. Eur. Thyroid J. 10, 185-197 (2021)

10. C. Zhang, J. Yin, C. Hu et al. Comparison of ultrasound guided percutaneous radiofrequency ablation and open thyroidectomy in the treatment of low-risk papillary thyroid microcarcinoma: a propensity score matching study. Clin. Hemorheol. Microcirc. Prepress, 1-9 https://doi.org/10.3233/CH-201087 (2021)

11. M. Zhang, R.P. Tufano, J.O. Russell et al. Ultrasound-guided radiofrequency ablation versus surgery for low-risk papillary thyroid microcarcinoma: results of over 5 years' follow-up. Thyroid 30, 408-417 (2020)

12. Q. Song, H. Gao, L. Ren et al. Radiofrequency ablation versus total thyroidectomy in patients with papillary thyroid microcarcinoma located in the isthmus: a retrospective cohort study. Int J. Hyperth. 38, 708-714 (2021)

13. L. Yan, M. Zhang, Q. Song et al. Ultrasound-guided radiofrequency ablation versus thyroid lobectomy for low-risk papillary thyroid microcarcinoma: a propensity-matched cohort study of 884 patients. Thyroid 31, 1662-1672 (2021)

14. J. Li, Y. Liu, J. Liu et al. Ultrasound-guided percutaneous microwave ablation versus surgery for papillary thyroid microcarcinoma. Int J. Hyperth. 34, 653-659 (2018)

15. W. Zhou, X. Ni, S. Xu et al. Ultrasound-guided laser ablation versus surgery for solitary papillary thyroid microcarcinoma: a retrospective study. Int J. Hyperth. 36, 897-904 (2019)

16. B. Xu, N.M. Zhou, W.T. Cao et al. Comparative study on operative trauma between microwave ablation and surgical treatment for papillary thyroid microcarcinoma. World J. Clin. Cases 6, 936-943 (2018)

17. J.F. Lin, P.K.C. Jonker, M. Cunich et al. Surgery alone for papillary thyroid microcarcinoma is less costly and more effective than long term active surveillance. Surgery 167, 110-116 (2020)

18. L. Hegedus, A. Miyauchi, R.M. Tuttle, Nonsurgical thermal ablation of thyroid nodules: not if, but why, when, and how? Thyroid 30, 1691-1694 (2020)

19. J. Chen, J. Cao, F. Qiu et al. The efficacy and the safety of ultrasound-guided ablation therapy for treating papillary thyroid microcarcinoma. J. Cancer 10, 5272-5282 (2019)

20. Y. Lan, Y. Luo, M. Zhang et al. Quality of life in papillary thyroid microcarcinoma patients undergoing radiofrequency ablation or surgery: a comparative study. Front Endocrinol. 11, 249 (2020)

21. Y.S. Lee, J.J. Jeong, K.H. Nam et al. Papillary carcinoma located in the thyroid isthmus. World J. Surg. 34, 36-39 (2010)

22. L. Rudmik, M. Drummond, Health economic evaluation: important principles and methodology. Laryngoscope 123, 1341-1347 (2013)

23. A. Vrachimis, I. Iakovou, E. Giannoula et al. Endocrinology in the time of COVID-19: Management of thyroid nodules and cancer. Eur. J. Endocrinol. 183, G41-g8 (2020)

24. S.R. Finlayson, J.D. Birkmeyer, Cost-effectiveness analysis in surgery. Surgery 123, 151-156 (1998)

25. J.A. Schwartz, S.D. Pearson, Cost consideration in the clinical guidance documents of physician specialty societies in the United States. JAMA Intern Med 173, 1091-1097 (2013)

26. J.P. Brito, I.D. Hay, J.C. Morris, Low risk papillary thyroid cancer. Bmj; 348, g3045 (2014)
27. B. Fautrel, A. Boonen, M. de Wit, et al. Cost assessment of health interventions and diseases. RMD Open 6, e001287 (2020).

28. J.C. Brand, M.J. Rossi, J.H. Lubowitz, Cost-effective medical care optimizes value over time. Arthroscopy 34, 2521-2523 (2018)

29. I.M. Rutkow, Socioeconomic aspects. World J. Surg. 23, 781-785 (1999)

30. A.M. Higgins, A.H. Harris, Health economic methods: costminimization, cost-effectiveness, cost-utility, and cost-benefit evaluations. Crit. Care Clin. 28, 11-24 (2012). v

31. Y. Min, X. Wang, H. Chen et al. Thermal ablation for papillary thyroid microcarcinoma: how far we have come? Cancer Manag. Res.;Vol. 12, 13369-13379 (2020)

32. L. Ji, Q. Wu, J. Gu et al. Ultrasound-guided percutaneous laser ablation for papillary thyroid microcarcinoma: a retrospective analysis of 37 patients. Cancer imaging 19, 16 (2019)

33. L. Yan, M. Zhang, Q. Song et al. The efficacy and safety of radiofrequency ablation for bilateral papillary thyroid microcarcinoma. Front Endocrinol. (Lausanne) 12, 663636 (2021)

34. L. Wang, D. Xu, Y. Yang et al. Safety and efficacy of ultrasoundguided percutaneous thermal ablation in treating low-risk papillary thyroid microcarcinoma: a pilot and feasibility study. J. Cancer Res Ther. 15, 1522-1529 (2019)

35. M. Zhang, Y. Luo, Y. Zhang et al. Efficacy and safety of ultrasound-guided radiofrequency ablation for treating low-risk papillary thyroid microcarcinoma: a prospective study. Thyroid 26, 1581-1587 (2016)

36. D.K. Teng, W.H. Li, J.R. Du et al. Effects of microwave ablation on papillary thyroid microcarcinoma: a five-year follow-up report. Thyroid 30, 1752-1758 (2020)

37. H.K. Lim, S.J. Cho, J.H. Baek et al. US-guided radiofrequency ablation for low-risk papillary thyroid microcarcinoma: efficacy and safety in a large population. Korean J. Radio. 20, 1653-1661 (2019)

38. X.J. Cao, M.A. Yu, Y.L. Zhu et al. Ultrasound-guided thermal ablation for papillary thyroid microcarcinoma: a multicenter retrospective study. Int J. Hyperth. 38, 916-922 (2021)

39. L. Yan, M. Zhang, Q. Song, F. Xie, Y. Luo Clinical outcomes of radio frequency ablation for multifocal papillary thyroid microcarcinoma versus unifocal papillary thyroid microcarcinoma: a propensity-matched cohort study. Eur. Radiol. 32, 1216-1226 (2022).

40. W. Zhou, S. Jiang, W. Zhan et al. Ultrasound-guided percutaneous laser ablation of unifocal T1N0M0 papillary thyroid microcarcinoma: preliminary results. Eur. Radio. 27, 2934-2940 (2017)

41. R. Valcavi, S. Piana, G.S. Bortolan et al. Ultrasound-guided percutaneous laser ablation of papillary thyroid microcarcinoma: a feasibility study on three cases with pathological and immunohistochemical evaluation. Thyroid 23, 1578-1582 (2013)

42. D.K. Teng, H.Q. Li, G.Q. Sui et al. Preliminary report of microwave ablation for the primary papillary thyroid microcarcinoma: a large-cohort of 185 patients feasibility study. Endocrine 64, 109-117 (2019)

43. R. Wu, Y. Luo, J. Tang et al. Ultrasound-guided radiofrequency ablation for papillary thyroid microcarcinoma: a retrospective analysis of 198 patients. Int J. Hyperth. 37, 168-174 (2020)

44. S. Bernardi, A. Palermo, R.F. Grasso et al. Current status and challenges of US-guided radiofrequency ablation of thyroid nodules in the long term: a systematic review. Cancers (Basel) 13, 2746 (2021).

45. S.J. Cho, S.M. Baek, H.K. Lim et al. Long-term follow-up results of ultrasound-guided radiofrequency ablation for low-risk papillary thyroid microcarcinoma: more than 5-year follow-up for 84 tumors. Thyroid 30, 1745-1751 (2020) 
46. Y. Zhu, Y. Che, S. Gao et al. Long-term follow-up results of PTMC treated by ultrasound-guided radiofrequency ablation: a retrospective study. Int. J. Hyperth. 38, 1225-1232 (2021)

47. R.P. Tufano, P. Pace-Asciak, J.O. Russell et al. Update of radiofrequency ablation for treating benign and malignant thyroid nodules. the future is now. Front Endocrinol. (Lausanne) 12, 698689 (2021)

48. D. Teng, G. Sui, C. Liu et al. Long-term efficacy of ultrasoundguided low power microwave ablation for the treatment of primary papillary thyroid microcarcinoma: a 3-year follow-up study. J. Cancer Res. Clin. Oncol. 144, 771-779 (2018)

49. B.R. Haugen, E.K. Alexander, K.C. Bible et al. 2015 American Thyroid Association Management Guidelines for Adult Patients with Thyroid Nodules and Differentiated Thyroid Cancer: The American Thyroid Association Guidelines Task Force on Thyroid Nodules and Differentiated Thyroid Cancer. Thyroid 26, 1-133 (2016) 